\title{
A Measurement of the Michel Parameters in Leptonic Decays of the Tau
}

R. Ammar, ${ }_{1}^{1}$ P. Baringer, ${ }^{1}$ A. Bean, ${ }^{1}$ D. Besson, ${ }^{1}$ D. Coppage,${ }^{1}$ C. Darling, ${ }^{1}$ R. Davis, ${ }^{1}$ N. Hancock, ${ }^{1}$ S. Kotov,${ }^{1}$ I. Kravchenko, ${ }^{1}$ N. Kwak, ${ }^{1}$ S. Anderson, ${ }^{2}$ Y. Kubota, ${ }^{2}$ M. Lattery, ${ }^{2}$ J. J. O'Neill, ${ }^{2}$ S. Patton, ${ }^{2}$ R. Poling, ${ }^{2}$ T. Riehle, ${ }^{2}$ V. Savinov, ${ }^{2}$ A. Smith, ${ }^{2}$ M. S. Alam, ${ }^{3}$ S. B. Athar, ${ }^{3}$ Z. Ling, ${ }^{3}$ A. H. Mahmood, ${ }^{3}$ H. Severini, ${ }^{3}$ S. Timm, ${ }^{3}$ F. Wappler, ${ }^{3}$ A. Anastassov, ${ }^{4}$ S. Blinov, ${ }^{4, *}$ J. E. Duboscq,${ }^{4}$ D. Fujino, ${ }^{4, \dagger}$ R. Fulton, ${ }^{4}$ K. K. Gan,${ }^{4}$ T. Hart, ${ }^{4}$ K. Honscheid, ${ }^{4}$ H. Kagan, ${ }^{4}$ R. Kass, ${ }^{4}$ J. Lee, ${ }^{4}$ M. B. Spencer, ${ }^{4}$ M. Sung, ${ }^{4}$ A. Undrus, ${ }^{4} *$ R. Wanke, ${ }^{4}$ A. Wolf,${ }^{4}$ M. M. Zoeller, ${ }^{4}$ B. Nemati, ${ }^{5}$ S. J. Richichi, ${ }^{5}$ W. R. Ross, ${ }^{5}$ P. Skubic,${ }^{5}$ M. Wood,${ }^{5}$ M. Bishai, ${ }^{6}$ J. Fast, ${ }^{6}$ E. Gerndt, ${ }^{6}$ J. W. Hinson, ${ }^{6}$ N. Menon, ${ }^{6}$ D. H. Miller, ${ }^{6}$ E. I. Shibata, ${ }^{6}$ I. P. J. Shipsey, ${ }^{6}$ M. Yurko, ${ }^{6}$ L. Gibbons, ${ }^{7}$ S. D. Johnson, ${ }^{7}$ Y. Kwon, ${ }^{7}$ S. Roberts, ${ }^{7}$ E. H. Thorndike, ${ }^{7}$ C. P. Jessop,${ }^{8}$ K. Lingel,${ }^{8}$ H. Marsiske, ${ }^{8}$ M. L. Perl, ${ }^{8}$ S. F. Schaffner, ${ }^{8}$ D. Ugolini, ${ }^{8}$ R. Wang, ${ }^{8}$ X. Zhou, ${ }^{8}$ T. E. Coan, ${ }^{9}$ V. Fadeyev,,${ }^{9}$ I. Korolkov, ${ }^{9}$ Y. Maravin,,${ }^{9}$ I. Narsky, ${ }^{9}$ V. Shelkov, ${ }^{9}$ J. Staeck, ${ }^{9}$ R. Stroynowski, ${ }^{9}$ I. Volobouev, ${ }^{9}$ J. Ye,${ }^{9}$ M. Artuso, ${ }^{10}$ A. Efimov,${ }^{10}$ F. Frasconi, ${ }^{10}$ M. Gao,${ }^{10}$ M. Goldberg,,${ }^{10}$ D. He, ${ }^{10}$ S. Kopp,${ }^{10}$ G. C. Moneti, ${ }^{10}$ R. Mountain, ${ }^{10}$ Y. Mukhin, ${ }^{10}$ S. Schuh, ${ }^{10}$ T. Skwarnicki, ${ }^{10}$ S. Stone,${ }^{10}$ G. Viehhauser,,${ }^{10}$ X. Xing, ${ }^{10}$ J. Bartelt, ${ }^{11}$ S. E. Csorna,${ }^{11}$ V. Jain, ${ }^{11}$ S. Marka, ${ }^{11}$ A. Freyberger, ${ }^{12}$ D. Gibaut, ${ }^{12}$ R. Godang, ${ }^{12}$ K. Kinoshita, ${ }^{12}$ I. C. Lai, ${ }^{12}$ P. Pomianowski, ${ }^{12}$ S. Schrenk, ${ }^{12}$ G. Bonvicini, ${ }^{13}$ D. Cinabro, ${ }^{13}$ R. Greene, ${ }^{13}$ L. P. Perera, ${ }^{13}$ B. Barish, ${ }^{14}$ M. Chadha, ${ }^{14}$ S. Chan, ${ }^{14}$ G. Eigen, ${ }^{14}$ J. S. Miller, ${ }^{14}$ C. O' Grady,${ }^{14}$ M. Schmidtler, ${ }^{14}$ J. Urheim, ${ }^{14}$ A. J. Weinstein, ${ }^{14}$ F. Würthwein, ${ }^{14}$ D. M. Asner, ${ }^{15}$ D. W. Bliss, ${ }^{15}$ W. S. Brower, ${ }^{15}$ G. Masek, ${ }^{15}$ H. P. Paar, ${ }^{15}$ J. Gronberg, ${ }^{16}$ R. Kutschke, ${ }^{16}$ D. J. Lange, ${ }^{16}$ S. Menary, ${ }^{16}$ R. J. Morrison, ${ }^{16}$ S. Nakanishi, ${ }^{16}$ H. N. Nelson, ${ }^{16}$ T. K. Nelson,,${ }^{16}$ C. Qiao, ${ }^{16}$ J. D. Richman, ${ }^{16}$ D. Roberts, ${ }^{16}$ A. Ryd, ${ }^{16}$ H. Tajima, ${ }^{16}$ M. S. Witherell, ${ }^{16}$ R. Balest, ${ }^{17}$ B. H. Behrens,${ }^{17}$ K. Cho, ${ }^{17}$ W. T. Ford, ${ }^{17}$ H. Park, ${ }^{17}$ P. Rankin, ${ }^{17}$ J. Roy, ${ }^{17}$ J. G. Smith, ${ }^{17}$ J. P. Alexander, ${ }^{18}$ C. Bebek, ${ }^{18}$ B. E. Berger,${ }^{18}$ K. Berkelman, ${ }^{18}$ K. Bloom,${ }^{18}$ D. G. Cassel,${ }^{18}$ H. A. Cho, ${ }^{18}$ D. M. Coffman, ${ }^{18}$ D. S. Crowcroft,${ }^{18}$ M. Dickson, ${ }^{18}$ P. S. Drell, ${ }^{18}$ K. M. Ecklund, ${ }^{18}$ R. Ehrlich, ${ }^{18}$ R. Elia, ${ }^{18}$ A. D. Foland, ${ }^{18}$ P. Gaidarev, ${ }^{18}$ R. S. Galik, ${ }^{18}$ B. Gittelman, ${ }^{18}$ S. W. Gray, ${ }^{18}$ D. L. Hartill, ${ }^{18}$ B. K. Heltsley, ${ }^{18}$ P. I. Hopman, ${ }^{18}$ S. L. Jones,${ }^{18}$ J. Kandaswamy,${ }^{18}$ N. Katayama, ${ }^{18}$ P.C. Kim, ${ }^{18}$ D. L. Kreinick, ${ }^{18}$ T. Lee, ${ }^{18}$ Y. Liu, ${ }^{18}$ G. S. Ludwig, ${ }^{18}$ J. Masui, ${ }^{18}$ J. Mevissen, ${ }^{18}$ N. B. Mistry, ${ }^{18}$ C. R. Ng, ${ }^{18}$ E. Nordberg, ${ }^{18}$ M. Ogg, ${ }^{18,}$ J. R. Patterson, ${ }^{18}$ D. Peterson, ${ }^{18}$ D. Riley, ${ }^{18}$ A. Soffer, ${ }^{18}$ C. Ward,${ }^{18}$ M. Athanas, ${ }^{19}$ P. Avery, ${ }^{19}$ C. D. Jones, ${ }^{19}$ M. Lohner, ${ }^{19}$ C. Prescott,${ }^{19}$ S. Yang, ${ }^{19}$ J. Yelton, ${ }^{19}$ J. Zheng, ${ }^{19}$ G. Brandenburg, ${ }^{20}$ R. A. Briere, ${ }^{20}$ Y. S. Gao, ${ }^{20}$ D. Y.-J. Kim,${ }^{20}$ R. Wilson, ${ }^{20}$ H. Yamamoto, ${ }^{20}$ T. E. Browder, ${ }^{21}$ F. Li,${ }^{21}$ Y. Li,,${ }^{21}$ J. L. Rodriguez, ${ }^{21}$ T. Bergfeld, ${ }^{22}$ B. I. Eisenstein, ${ }^{22}$ J. Ernst, ${ }^{22}$ G. E. Gladding, ${ }^{22}$ G. D. Gollin, ${ }^{22}$ R. M. Hans, ${ }^{22}$ E. Johnson, ${ }^{22}$ I. Karliner, ${ }^{22}$ M. A. Marsh, ${ }^{22}$ M. Palmer, ${ }^{22}$ M. Selen, ${ }^{22}$ J. J. Thaler, ${ }^{22}$ K. W. Edwards, ${ }^{23}$ A. Bellerive, ${ }^{24}$ R. Janicek, ${ }^{24}$ D. B. MacFarlane, ${ }^{24}$ K. W. McLean, ${ }^{24}$ P. M. Patel,,${ }^{24}$ and A. J. Sadoff ${ }^{25}$

(CLEO Collaboration)

\author{
${ }^{1}$ University of Kansas, Lawrence, Kansas 66045 \\ ${ }^{2}$ University of Minnesota, Minneapolis, Minnesota 55455 \\ ${ }^{3}$ State University of New York at Albany, Albany, New York 12222 \\ ${ }^{4}$ Ohio State University, Columbus, Ohio 43210 \\ ${ }^{5}$ University of Oklahoma, Norman, Oklahoma 73019 \\ ${ }^{6}$ Purdue University, West Lafayette, Indiana 47907 \\ ${ }^{7}$ University of Rochester, Rochester, New York 14627 \\ ${ }^{8}$ Stanford Linear Accelerator Center, Stanford University, Stanford,California 94309 \\ ${ }^{9}$ Southern Methodist University, Dallas, Texas 75275 \\ ${ }^{10}$ Syracuse University, Syracuse, New York 13244 \\ ${ }^{11}$ Vanderbilt University, Nashville, Tennessee 37235 \\ ${ }^{12}$ Virginia Polytechnic Institute and State University, Blacksburg, Virginia 24061 \\ ${ }^{13}$ Wayne State University, Detroit, Michigan 48202 \\ ${ }^{14}$ California Institute of Technology, Pasadena, California 91125 \\ ${ }^{15}$ University of California, San Diego, La Jolla, California 92093 \\ ${ }^{16}$ University of California, Santa Barbara, California 93106 \\ ${ }^{17}$ University of Colorado, Boulder, Colorado 80309-0390 \\ ${ }^{18}$ Cornell University, Ithaca, New York 14853 \\ ${ }^{19}$ University of Florida, Gainesville, Florida 32611 \\ ${ }^{20}$ Harvard University, Cambridge, Massachusetts 02138 \\ ${ }^{21}$ University of Hawaii at Manoa, Honolulu, Hawaii 96822 \\ ${ }^{22}$ University of Illinois, Champaign-Urbana, Illinois 61801 \\ ${ }^{23}$ Carleton University, Ottawa, Ontario, Canada KIS $5 B 6$ and the Institute of Particle Physics, Canada
}




\section{${ }^{24}$ McGill University, Montréal, Québec, Canada H3A 2 T8 and the Institute of Particle Physics, Canada \\ ${ }^{25}$ Ithaca College, Ithaca, New York 14850}

(Received 26 December 1996)

We have measured the spectral shape Michel parameters $\rho$ and $\eta$ using leptonic decays of the $\tau$, recorded by the CLEO II detector. Assuming $e-\mu$ universality in the vectorlike couplings, we find $\rho_{e \mu}=0.735 \pm 0.013 \pm 0.008$ and $\eta_{e \mu}=-0.015 \pm 0.061 \pm 0.062$, where the first error is statistical and the second systematic. We also present measurements for the parameters for $e$ and $\mu$ final states separately. [S0031-9007(97)03312-7]

PACS numbers: 13.35.Dx, 14.60.Fg, 14.80.Cp

Leptonic $\tau$ decays are sensitive probes of the charged weak interaction since the strong interaction plays no role in these decays. The Lorentz structure of the $\mu W \nu_{\mu}$ and $e W \nu_{e}$ currents is well established. In this paper, we investigate the $\tau W \nu_{\tau}$ vertex.

In the decays of the $\tau$ to $\ell \nu \bar{\nu}$, information on the decay can be extracted from the shape of the momentum distribution of the lepton $\ell$, and from its angular distribution relative to the parent $\tau$ spin direction [1-4]. After integration over the unobserved neutrino momenta and the spin of $\ell$, and neglecting radiative effects we can write the charged lepton momentum spectrum as

$$
\frac{1}{\Gamma} \frac{d \Gamma}{d x}=x^{2} \frac{\left[12(1-x)+\frac{4 \rho}{3}(8 x-6)+24 \eta \frac{m_{\ell}}{m_{\tau}} \frac{(1-x)}{x}\right]}{1+4 \eta\left(m_{\ell} / m_{\tau}\right)},
$$

where $\rho$ and $\eta$ are the spectral shape Michel parameters [1], and $x=E_{\ell} / E_{\max }$ is the lepton energy scaled to the maximum energy $E_{\max }=\left(m_{\tau}^{2}+m_{\ell}^{2}\right) / 2 m_{\tau}$ in the $\tau$ rest frame. In the standard model (SM), $\rho=3 / 4$ and $\eta=0$. Since $\tau^{+} \tau^{-}$pairs are produced with no net polarization at $e^{+} e^{-}$center-of-mass energies below the $Z^{0}$ mass, this spectrum is not sensitive to the spin-dependent parameters $\xi$ and $\delta$.

Ignoring scalar and tensor interactions, $\rho \neq 3 / 4$ suggests the mixing of right-handed and left-handed vector currents $[4,5]$. Assuming that the $V-A$ coupling is dominant, $\eta \neq 0$ suggests the presence of a scalar boson that couples to a right-handed $\tau$ and a right-handed $\ell$. Interference between the amplitudes of this scalar boson and the SM $W_{L}$ boson alters the low momentum region of the $\mu$ spectrum. This effect is helicity suppressed in the $e$ spectrum since it corresponds to a flipping of the lepton's spin, and therefore scales with the lepton mass. In the two-Higgs-doublet model with a scalar charged Higgs boson, one would have, in the decay $\tau \rightarrow \mu \nu \bar{\nu}$, $\eta_{\mu}=-\left(m_{\tau} m_{\mu} \tan ^{2} \beta\right) / 2 m_{H}^{2}$ [6], where $\beta$ is the ratio of the vacuum expectation values of the neutral components of the two Higgs doublets, and $m_{H}$ is the Higgs boson mass.

The data used in this analysis were produced in the $e^{+} e^{-} \rightarrow \tau^{+} \tau^{-}$reaction at the Cornell electron storage ring CESR, operating at $E_{\mathrm{cm}} \simeq 10.6 \mathrm{GeV}$, and collected with the CLEO II detector [7]. Using $3500 \mathrm{pb}^{-1}(3.20 \times$ $10^{6}$ produced $\tau$ pairs) of analyzed data, we measure $\rho_{e}$ using the $e \nu \nu$ mode, and $\rho_{\mu}$ and $\eta_{\mu}$ using the $\mu \nu \nu$ mode. Adding the assumption of $e-\mu$ universality we also analyze the two modes simultaneously to measure $\rho_{e \mu}$ and $\eta_{e \mu}$.
To isolate a pure sample, we select events in which the $\tau^{-}$has decayed to $e \nu \bar{\nu}$ or $\mu \nu \bar{\nu}$ (signal) and the recoiling $\tau^{+}$has decayed to $h^{+} \pi^{0} \bar{\nu}_{\tau}$ (tag) [8], where $h$ refers to a charged $\pi$ or $K$. The $h^{+} \pi^{0} \bar{\nu}_{\tau}$ mode is chosen because of its large branching fraction, and because events so tagged are easily distinguishable from QED and other non- $\tau$ backgrounds.

Events are required to have two oppositely charged good tracks in the barrel region of the detector $|\cos \theta|<0.71$, where $\theta$ is the polar angle of the track with respect to the beam axis. We consider pairs of barrel photons, above $100 \mathrm{MeV}$ each, as $\pi^{0}$ candidates if their invariant mass lies within 3 standard deviations $\left(\sigma_{\gamma \gamma}=5-9 \mathrm{MeV}\right)$ of the $\pi^{0}$ mass.

The track further away in angle from the reconstructed $\pi^{0}$ is required to be either an $e$ or $\mu$. Electrons above $0.5 \mathrm{GeV} / c$ are identified by momentum and specific ionization measurements from the tracking systems, and energy measurements from the electromagnetic calorimeter. Muons above $1.5 \mathrm{GeV} / c$ are identified by projecting tracks to hits in muon counters beyond at least three absorption lengths of material. Lepton identification efficiencies and fake rates are determined from the data.

Cuts are employed to suppress $e e(\gamma)$ and $\mu \mu$ events. We allow no more than one identified $e$. Remaining backgrounds from these sources, along with those due to $2 \gamma$ annihilation, cosmic rays, beam gas interactions, and $q \bar{q}$ production [including $\Upsilon(4 S) \rightarrow B \bar{B}$ ] are all estimated to be negligible. Events with isolated photonlike unused showers above $75 \mathrm{MeV}(100 \mathrm{MeV})$ in the barrel (endcap) are rejected to reduce background from multi- $\pi^{0}$ modes mimicking the tag mode.

We identify 31568 [21766] $e$ 's [ $\mu$ 's] with an estimated misidentification background of $(0.178 \pm 0.026) \%$ $[(1.08 \pm 0.16) \%]$. The number of observed events is consistent with expectations from world average branching fractions. No correction is made for the small fake electron contamination. The product of the momentum dependent fake rate (determined using the $h^{ \pm}$from tag decay), and the parent hadron distribution (obtained by discarding all identified $e$ and $\mu$ events on the signal side), estimates the fake muon spectrum which is subtracted from the signal spectrum. Using Monte Carlo (MC) simulation, we predict a remnant multi- $\pi^{0}$ background contamination of $(1.19 \pm 0.05) \%[(1.38 \pm 0.06) \%]$ of the tag decays accompanying the electronic [muonic] decays. 


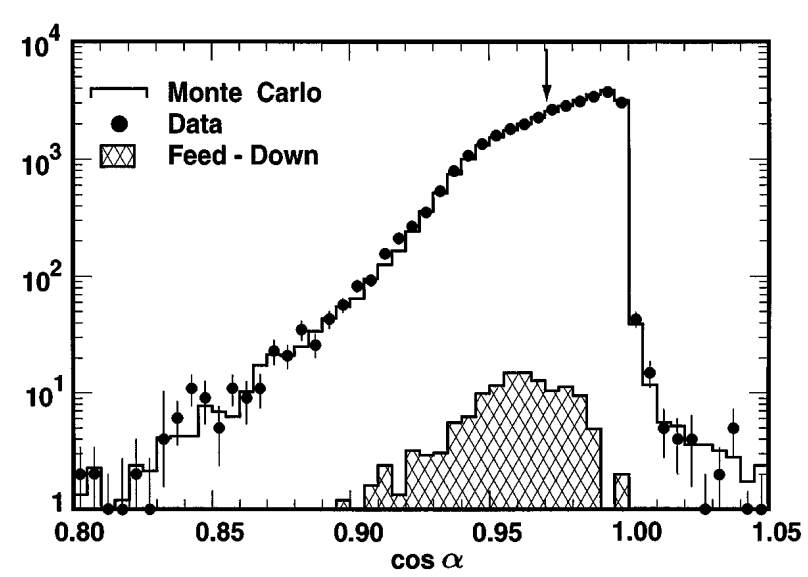

FIG. 1. The distribution of $\cos \alpha$ for the data (dots), the generic $\tau$ MC simulation (solid histogram), and the background contamination from multi- $\pi^{0}$ decay modes (hatched region). The arrow indicates the nominal minimum requirement. Events with $\cos \alpha>1$ result from measurement errors and are discarded.

The ideal Lorentz frame for measuring $\rho$ and $\eta$ simultaneously is the $\tau$ rest frame. The lepton spectrum observed in the laboratory (LAB) differs from the $\tau$ rest frame spectrum due to the Lorentz boost. All sensitivity to $\eta$ is now restricted to the low momentum region for which muon identification at CLEO is limited. The rest frame lepton spectrum cannot be measured since the unobserved neutrinos in these events preclude the explicit reconstruction of the entire event. One can, however, utilize information from the tag $\tau$ to estimate a pseudo rest frame (PRF) of the lepton's parent $\tau$ [9]. In the absence of radiation, the two $\tau$ 's are produced back-to-back, and at the beam energy. We select events in which the direction of the tag $h \pi^{0}$ system reliably estimates the flight direction of the parent $\tau$. In the decay $\tau \rightarrow A \nu$, where $A$ is the hadronic system and $\alpha$ is the angle between the $\tau$ and $A$ momenta in the LAB frame, we have $m_{\tau}^{2}+m_{A}^{2}-2 E_{\tau} E_{A}+2 p_{\tau} p_{A} \cos \alpha=m_{\nu}^{2} . E_{A}, p_{A}$, and $m_{A}$ are all measured quantities, and we assume $m_{\nu}=0$ and $E_{\tau}=E_{\text {beam }}$ to calculate $\cos \alpha$. For $\cos \alpha \simeq 1$, the tag $h \pi^{0}$ momentum gives an excellent approximation of the parent $\tau$ direction. Accordingly, we select events with $\cos \alpha \geq 0.970$ (Fig. 1) and reconstruct the PRF spectrum. After the $\cos \alpha$ requirement, one retains 18587 (12580) electrons (muons).

In the PRF, we include 2931 muons that lie between $0.5-1.5 \mathrm{GeV} / c$ in LAB momentum, below the efficiency

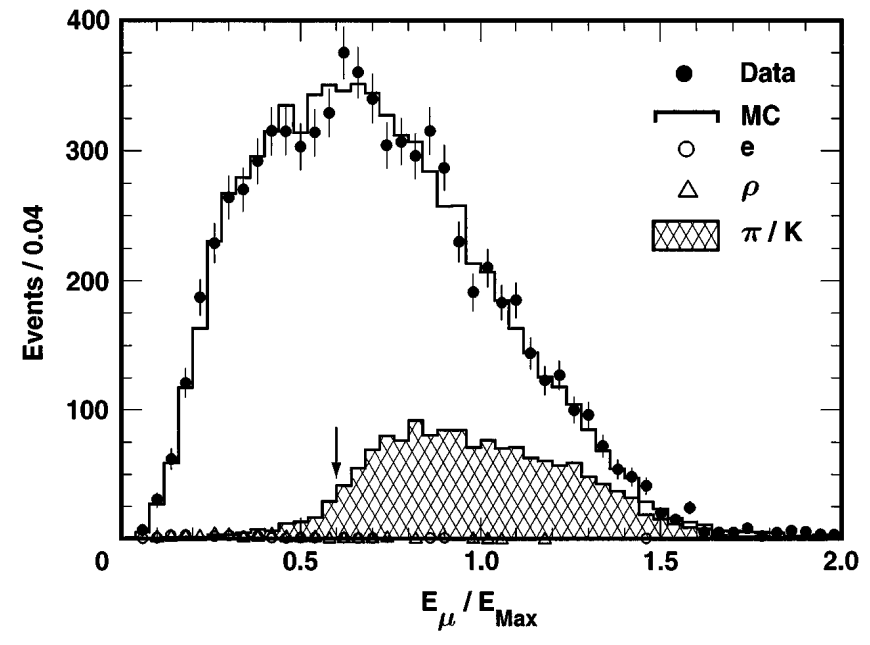

FIG. 2. The scaled PRF energy spectrum in the data (dots) and the MC simulation (solid histogram), for $\mu$ 's identified kinematically. The MC spectra for the background modes are also illustrated; their contributions are small for $E_{\mu} / E_{\max }<$ 0.6 , indicated by the arrow.

plateau of the muon identification system. These muons are identified by elimination of all other possible decay hypotheses $\left(\pi \nu, K \nu, e \nu \bar{\nu}\right.$, and $\left.h n \pi^{0} \nu\right)$. We define $X=E_{\mu} / E_{\max }$ as the PRF muon energy scaled to the maximum possible energy $E_{\max }=\left(m_{\mu}^{2}+m_{\tau}^{2}\right) / 2 m_{\tau}$ in the $\tau$ rest frame (Fig. 2). For the two-body modes $\pi \nu$ and $K \nu$, we have $X_{\pi(K)}=0.89(0.95)$ in the true rest frame. A cut at $X \leq 0.6$ reduces the $\pi / K$ contamination to $(2.63 \pm 0.21) \%$. The $p_{\mu} \geq 0.5 \mathrm{GeV} / c$ requirement, along with an explicit electron veto reduces the electron contamination to $(0.64 \pm 0.11) \%$. No extra unmatched showers above $60 \mathrm{MeV}$ are allowed, either in the barrel or the endcap region, to minimize backgrounds from $h \pi^{0} \nu$ and other multi- $\pi^{0}$ modes; this contamination is estimated to be $(0.78 \pm 0.12) \%$. These backgrounds are calculated with the MC simulation and subtracted from the data. Thus, we recover a small but pure sample of muons particularly sensitive to the $\eta$ parameter.

The prediction $d N_{\text {obs }} / d x$ for the charged daughter lepton spectrum, integrated over the two undetected neutrino momenta, and averaged over the $\tau$ helicity, can be expressed in terms of three MC spectra $d N / d x[\rho, \eta]$ : the standard $V-A[3 / 4,0]$ spectrum, the $V+A[0,0]$ spectrum and the $\eta=1[3 / 4,1]$ spectrum. With each reconstructed MC spectrum normalized to the total number of events, the data spectrum is represented by

$$
\begin{aligned}
S \frac{d N_{\mathrm{obs}}}{d x}[\rho, \eta]= & \left\{\frac{(4 \rho / 3-\eta)\left(\epsilon_{\mathrm{V}-\mathrm{A}}\right)}{1+4 \eta\left(m_{\ell} / m_{\tau}\right)}\right\} \frac{d N}{d x}[3 / 4,0] \\
& +\left\{\frac{(1-4 \rho / 3)\left(\epsilon_{\mathrm{V}+\mathrm{A}}\right)}{1+4 \eta\left(m_{\ell} / m_{\tau}\right)}\right\} \frac{d N}{d x}[0,0]+\left\{\frac{\eta\left[1+4\left(m_{\ell} / m_{\tau}\right)\right]\left(\epsilon_{\eta=1}\right)}{1+4 \eta\left(m_{\ell} / m_{\tau}\right)}\right\} \frac{d N}{d x}[3 / 4,1],
\end{aligned}
$$

where $S$ is the sum of the three coefficients $(\{\})$. The lepton momentum cutoff results in a different average efficiency $\epsilon$ for each MC spectrum; a small efficiency correction is required. By construction, the fit function integrates to the total number of observed events for all physical values of the parameters. 
We perform a $\chi^{2}$ fit of the data spectrum to the above function of three binned MC spectra. We use the KORALB(v2.2) [10], TAUOLA(v2.4) [11], and PHOTOS(v1.06) [12] MC packages to model the production and decay of $\tau$ pairs, and the GEANT 3.15 [13] program to simulate the response of the CLEO II detector. Small modifications to the TAUOLA package were required to generate decays with non-SM values of $\rho$ and $\eta$ [14].
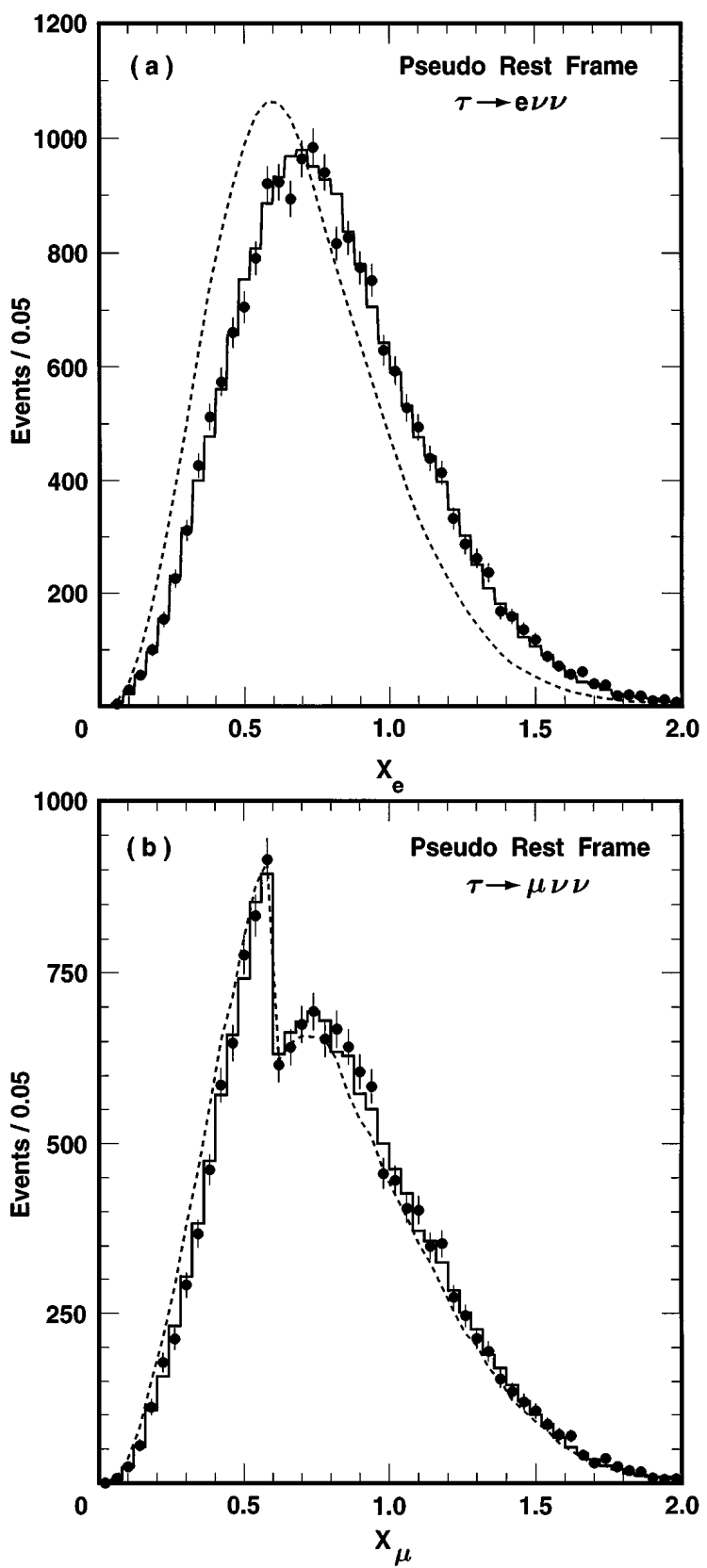

FIG. 3. The (a) $e$, (b) $\mu$ scaled PRF energy spectra with the data (dots) and fit function (solid histogram). The dotted line in (a) represents the electron $V+A$ MC spectrum and the dotted line in (b) represents the muon $\eta=1 \mathrm{MC}$ spectrum. Events with $X>1$ result from the imperfect reconstruction of the $\tau$ direction. The addition of the low momentum muons results in the discontinuity observed at $X_{\mu}=0.6$ in (b).
All effects due to radiation, resolution, and efficiency are included in the three MC spectra.

Events which survive the $\cos \alpha$ constraint are analyzed in the PRF (Fig. 3), the remainder being analyzed in the $\mathrm{LAB}$ frame. In the electronic decay mode, only the first two terms in the previous equation are relevant. We use a weighted average of the two independent frame results to measure $\rho_{e}=0.732 \pm 0.014$ with a $\chi^{2} /$ d.o.f. of $51.5 / 46$ (36.2 /44) in the PRF (LAB frame). In the muonic decay mode, the parameters $\rho_{\mu}$ and $\eta_{\mu}$ are strongly correlated and are simultaneously measured. The weighted average yields $\rho_{\mu}=0.747 \pm 0.048$ and $\eta_{\mu}=0.010 \pm 0.149$, with a $\chi^{2} /$ d.o.f. of $26.9 / 34(28.1 / 33)$ in the PRF (LAB frame). The correlation coefficient $C_{\rho \eta}$ is 0.949 . Omission of the low momentum muons, recovered without conventional muon identification results in significantly larger errors. Results obtained on analyzing the two frames of reference independently are consistent with each other; the errors in the LAB frame are twice as large.

The high precision on $\rho$ obtained in the electron mode analysis is now used to constrain the $\eta$ parameter in a simultaneous fit to both modes. Assuming lepton universality in the vectorlike couplings, and no tensor interactions, we constrain $\rho_{e}=\rho_{\mu}$. We use $\eta_{e \mu}$ to denote the value of $\eta_{\mu}$ measured with this constraint. No constraint is placed on $\eta_{e}$ since we are insensitive to it. We measure $\rho_{e \mu}^{\tau}=0.735 \pm 0.013, \eta_{e \mu}^{\tau}=-0.015 \pm 0.061$, $C_{\rho \eta}=0.614$ with a $\chi^{2} /$ d.o.f. of $69.5 / 75(62.7 / 78)$ in the PRF (LAB frame). Again the two frames yield consistent results. We show in Fig. 4 results of the combined mode fit along with the measurements from the individual modes.

Table I lists the systematic error contributions from all dominant sources. Although the MC samples are each a factor of 10 times larger than the data, MC statistics

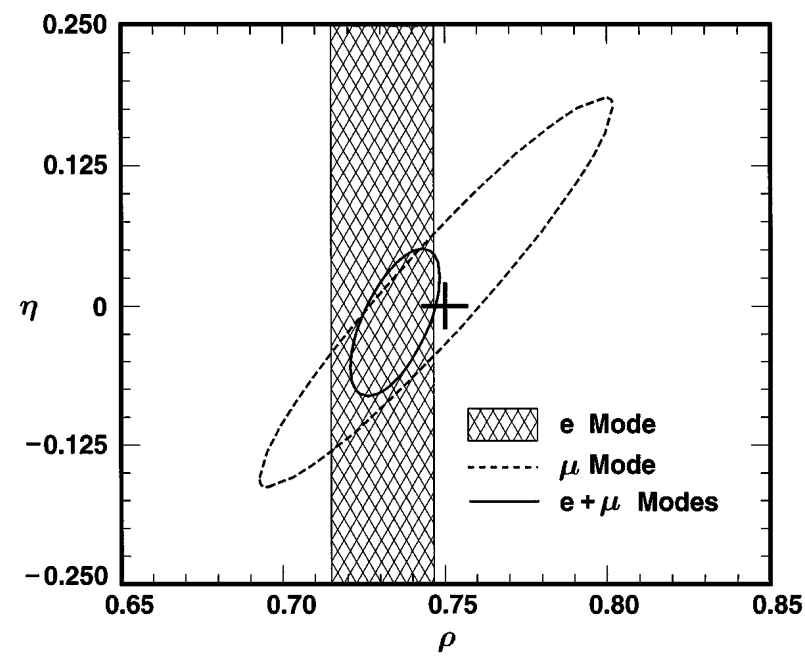

FIG. 4. The shaded band denotes the $e$ mode result; the dotted (solid) ellipse indicates the $\mu$ mode ( $e$ and $\mu$ combined) $1 \sigma$ error ellipse obtained in the $\eta-\rho$ plane. The SM expectation is given by the cross. 
TABLE I. All significant sources of errors.

\begin{tabular}{lcccrc}
\hline \hline \multicolumn{1}{c}{ Source } & $\rho_{e}$ & $\rho_{\mu}$ & $\eta_{\mu}$ & $\rho_{e \mu}$ & $\eta_{e \mu}$ \\
\hline Electron ID & $<0.001$ & $\ldots$ & $\ldots$ & $<0.001$ & 0.001 \\
Muon ID & $\ldots$ & 0.004 & 0.018 & 0.001 & 0.024 \\
Fake electron & 0.004 & $\ldots$ & $\ldots$ & 0.004 & 0.015 \\
Fake muon & $\ldots$ & 0.025 & 0.106 & 0.002 & 0.025 \\
Feed-down & 0.001 & 0.002 & 0.007 & 0.002 & 0.008 \\
Trigger & 0.002 & 0.006 & 0.019 & 0.002 & 0.005 \\
Bin migration & 0.001 & 0.020 & 0.066 & 0.002 & 0.019 \\
Correlations & 0.003 & 0.012 & 0.050 & 0.003 & 0.035 \\
Radiation & 0.005 & 0.003 & 0.050 & 0.001 & 0.004 \\
MC statistics & 0.005 & 0.026 & 0.089 & 0.005 & 0.026 \\
Total systematics & 0.009 & 0.044 & 0.171 & 0.008 & 0.062 \\
Data statistics & 0.014 & 0.048 & 0.149 & 0.013 & 0.061 \\
\hline \hline
\end{tabular}

remains one of the largest sources of systematic error. The complete CLEO MC simulation is used to determine the efficiencies of all applied cuts, along with their effects on the lepton momentum distributions; independent data samples are used to calibrate the MC simulation wherever possible.

Lepton identification efficiencies are measured from the data. Statistical uncertainties in these measurements lead to small systematic errors. The MC estimates for the trigger efficiencies are close to $100 \%$ in both modes. The absolute trigger efficiency measured in the data varied by a significant amount during the data collection, but these changes are momentum independent. To estimate the systematic contributions from background sources, both the normalization and the momentum dependence of each source are varied within allowed ranges. High momentum tracks can be mismeasured by as much as $50-$ $100 \mathrm{MeV} / c$, and the resulting bin migration alters the observed spectrum shape. All the fits performed utilize $100 \mathrm{MeV} / c$ bins and the parameters are determined with a large range of bin sizes to evaluate systematic contributions.

Detector and global cuts, spin correlations, and radiation all correlate the signal and tag $\tau$ decays in the event. These effects are all modeled in the MC, and we study systematic errors arising from their imperfect modeling by calculating the parameters using reweighted MC spectra. The weights are calculated from data and MC simulation comparisons of the $\pi^{0}$ energy spectrum, the $\pi^{ \pm}$momentum spectrum, and the distribution of the angle between these two pions. Variations of as much as 3 statistical standard deviations are used for the weights.

TABLE II. Results obtained in this analysis along with the corresponding previous world average results.

\begin{tabular}{ccc}
\hline \hline Parameter & This result & World average \\
\hline$\rho_{e}^{\tau}$ & $0.732 \pm 0.014 \pm 0.009$ & $0.736 \pm 0.028$ \\
$\rho_{\mu}^{\tau}$ & $0.747 \pm 0.048 \pm 0.044$ & $0.74 \pm 0.04$ \\
$\eta_{\mu}^{\tau}$ & $0.010 \pm 0.149 \pm 0.171$ & $-0.24 \pm 0.29$ \\
$\rho_{e \mu}^{\tau}$ & $0.735 \pm 0.013 \pm 0.008$ & $0.742 \pm 0.027$ \\
$\eta_{e \mu}^{\tau}$ & $-0.015 \pm 0.061 \pm 0.062$ & $-0.01 \pm 0.14$ \\
\hline \hline
\end{tabular}

We evaluate the radiation systematic error by varying the radiation prediction in the MC spectrum by as much as $\pm 10 \%$ in the fits performed to extract the two parameters. Initial and final state radiation have the largest rate and the greatest potential to distort the momentum spectrum; the contribution from decay radiation photons in $\tau \rightarrow \ell \nu \nu \gamma$, and photons resulting from external bremsstrahlung in the detector material are negligible.

MC generator-level tests, without any detector simulation, confirm that the fit procedure tracks both parameters over their respective allowed ranges in parameter space; no systematic bias is associated with the fitting procedure.

The results obtained for the different parameters measured, along with the previous world average measurements [15], are presented in Table II.

In conclusion, all results are consistent with previous measurements, and with the $V-A$ theory. They are more precise than all previous measurements, and help constrain the new world average results considerably. This measurement of the $\eta$ parameter provides a lower limit on the charged Higgs mass: $M_{H^{ \pm}}>(0.97 \times \tan \beta) \mathrm{GeV}$ at the $90 \%$ confidence level.

We gratefully acknowledge the effort of the CESR staff in providing us with excellent luminosity and running conditions. This work was supported by the National Science Foundation, the U.S. Department of Energy, the Heisenberg Foundation, the Alexander von Humboldt Stiftung, Research Corporation, the Natural Sciences and Engineering Research Council of Canada, and the A.P. Sloan Foundation.

*Permanent address: BINP, RU-630090 Novosibirsk, Russia.

†Permanent address: Lawrence Livermore National Laboratory, Livermore, CA 94551.

*Permanent address: University of Texas, Austin, TX 78712.

[1] L. Michel, Proc. Phys. Soc. London A 63, 514 (1950).

[2] T. Kinoshita and A. Sirlin, Phys. Rev. 108, 844 (1957).

[3] C. Bouchiat and L. Michel, Phys. Rev. 106, 170 (1957).

[4] F. Scheck, Phys. Rep. 44, 187 (1978).

[5] W. Fetscher, Phys. Rev. D 42, 1544 (1990).

[6] A. Stahl, Phys. Lett. B 324, 121-124 (1994).

[7] CLEO Collaboration, Y. Kubota et al., Nucl. Instrum. Methods Phys. Res., Sect. A 320, 66 (1992).

[8] Charge conjugate processes are implied.

[9] ARGUS Collaboration, H. Albrecht et al., Phys. Lett. B 341, 441-447 (1995).

[10] S. Jadach and Z. Was, Comput. Phys. Commun. 36, 191 (1985).

[11] S. Jadach, J. H. Kuhn, and Z. Was, Report No. CERNTH-5856/90, 1990.

[12] E. Barberio, B. van Eijk, and Z. Was, Report No. CERNTH-5857/90, 1990.

[13] R. Brun et al., Report No. CERN dd/ee/84-1.

[14] M. Schmidtler, Report No. IEKP-KA/93-14, 1993.

[15] R. M. Barnett et al., Particle Data Group, Phys. Rev. D 54, 1 (1996). 